Article

\title{
A Perspective on the Next-Generation Forest Register in the Era of GIS
}

\author{
Kazuhiro Tanaka *
}

\begin{abstract}
This paper points out some defects of the current forest register and proposes a concept of next-generation forest register from the view point of GIS application to forest management. Since the fundamental purpose of the GIS is to conduct spatial analysis of multiple thematic maps, one of the basic requirements for the next-generation forest register is the management of forest information on a thematic map basis by using the spatial analysis function of the forest GIS. The main proposals are as follows. The forest register information should be divided into multiple pieces according to thematic maps and their attribute information should be managed separately. The administrative data is an accumulation of thematic maps created every year. Data on forest management should be shared with forestry cooperatives and updated by forestry cooperatives electronically. The issue of personal information can be resolved by dividing the forest register information and managing it separately. Detailed historical information of stands is not necessarily contained in the database; rather, utilizing hyperlinks to the Forest Karte (record). The minimum information of forest GIS may be the thematic map of the forest physiognomy. A forestry experiment station-type forest GIS which is equipped with advanced spatial analysis functions is needed, and analysis and research results obtained from the full use of these functions are provided to the forest GIS used by administrative offices and forestry cooperatives.
\end{abstract}

Keywords: forest register, GIS, spatial analysis, thematic map

\section{INTRODUCTION}

All prefectures have already introduced the forest GIS in Japan, and staffs of not only prefectures but also forestry cooperatives are now starting to use forest GIS. The forest GIS will be recognized as an indispensable system for forest management in the near future. With the spread of the forest GIS, both usage of forest GIS and needs for forest GIS have been changing. It is getting clear that there are new problems on the effective use of forest GIS such as how to gather and update the attribute information, how to apply the function of spatial analysis, how to provide the fundamental thematic maps of forest GIS to users. In addition, we are still having some difficulty in discovering how an ideal next-generation forest register should be. Here, forest register is an account book for forest management in which present condition and related information of each forest stand are recorded.

In this paper, I point out some defects of the current forest register and propose a concept of next-generation

\footnotetext{
- Graduate School of Life and Environmental Sciences, Kyoto Prefectural University 606-8522 Kyoto Japan E-mail: tanakazu@kpu.ac.jp(K. Tanaka)
}

forest register from the view point of GIS application to forest management.

\section{DEFECTS IN THE CURRENT FOREST REGISTER}

The forest register prepared by prefectures is used as attribute information for the forest GIS. However, the forest register has some structural defects as explained below.

First, the forest register originally designed to show the current state of forest stands. However, the attribute information used for the present-day forest GIS should be more than just its current state. A next-generation forest register which includes the management history needs to be established.

Second, the gap between the information in the forest register and actual stand condition is growing larger, and that gap is sometimes too big to be ignored. For example, the register says there is an area of artificial forest in a certain place but in fact there is only a poor afforested land where trees do not grow well, and there are numerous cases such as this. Accurate information should be prepared in the forest register in order to make efficient use of the register.

Third, the forest register focuses on information about timber production and includes little information about various public benefits that the forests provide. Workers in the field need information about how the forest functions in a way that 
preserves water and protects the soil, as well as in maintaining biodiversity and the ecosystem, and furthermore, they need information about damage to wildlife and damage by disease or insect pests. It is expected that forests features which provide public benefits should be fully used, but the contents of the current forest register are definitely not enough. A nextgeneration forest register needs to be created.

Fourth, administrative offices and on-site workers do not agree on how to use the forest register and when it should be updated. Some prefectures believe the forest register should only be updated every five years, but forestry cooperatives need to update the forest register more frequently depending on how the forest is managed. Old information does not help them use the forest GIS effectively.

Lastly, the forest register cannot be disclosed because it contains some personal information. This causes another problem, that is, manufactures in the log production industry, who support local forestry activities, cannot obtain the required information. This also obstructs the introduction of healthy competition in the local log production marketplace.

The above-mentioned issues about the current forest register should be solved in order to establish a nextgeneration forest register that uses GIS as its core and a system that enables continuous updating of data as soon as possible.

\section{DEVELOPING PHASES OF THE FOREST GIS}

So far, the forest GIS is thought to have five developing phases.

The first-generation GIS is a system where subcompartment polygons are created and the polygons are linked to the information from the forest register, which is used as attribute information. Most forest GISs used by prefectures are first-generation.

The second-generation forest GIS is a system where vector data created from existing geographic information such as paper maps are input, and the GIS analyzes that information to make a new thematic map according to the object. This is used when a forest function assessment sectional map based on existing materials is created, for example.

The third-generation forest GIS can analyze monitoring results such as remote sensing information and new forest information, and the fourth-generation forest GIS uses the Internet to collect information about local forests and provides a system to share such information with locals who are involved in the issues.

The fifth-generation forest GIS can analyze various types of forest information collectively and conduct scenario analyses according to possible future events so that the results can be applied to policy making and consensus building.

The first-generation forest GIS is just information of the forest register used as attribute information for subcompartment polygons, and this simply makes it easier to display and search for forest information and create sectional maps. In addition, the information contained in the forest register is basically the current forest state, so there still remains the issue of maintenance and accumulation methods for each stand's history.
As the fifth-generation forest GIS is used for policy making and consensus building, this type of forest GIS is required to collect various types of information from external sources and make thematic maps or materials according to every single request. Accordingly, we have to review what kind of attribute information should be installed to the forest GIS as the minimum level of information necessary. Also, the forest register must have a flexible structure so that any external data can be taken into the register in various formats.

\section{FUNCTIONS OF THE FOREST GIS}

Before thinking about the next-generation forest register based on the forest GIS, we should understand the characteristics of GIS functions to build a structure that allows full use of the GIS functions. This is why we have to reconsider what functions the GIS provide.

The GIS generally provides the following four functions: database function, spatial analysis function, information sending and sharing function, and decision-making support function. Among these functions, the most important function that is essential to the GIS is the spatial analysis function (TANAKA and YoshIDA, 2005).

Some people think the GIS is a database with map information, which is not correct. Since those who only know the first-generation forest GIS are told that the forest GIS is a system where sub-compartment polygons linked to the information from the forest register, which is used as attribute information, this misunderstanding may be inevitable. However, this way of understanding is only about a rough view of the way the next-generation forest register should be.

Think about how to hold geographic information with the GIS, and compare that with a paper map. One commercial paper map shows all types of geographic information on it. However, the GIS does not hold geographic information in that way. The GIS creates different geographic information for every thematic map and keeps it as a file. For example, it creates a thematic map of a water system consisting of information about rivers and other inland waters, or it creates a thematic map of road networks, collecting geographic information only on roads and streets. After that, it calls only thematic maps needed for the analysis, and conducts spatial analysis dealing with these maps as a layer (Fig. 1). Information such as a paper map on the market is used for background images as raster data.

The key to the GIS is the spatial analysis using multiple thematic maps. Each thematic map has its own attribute information, and by using an overlay function called union, such attribute information is automatically inherited to finer polygons created by the overlay.

\section{POINT DATA WITH GPS INFORMATION, THE MAIN STREAM OF NEW FOREST INFORMATION: CALCULATION OF PLANES FROM POINTS}

When thinking about the way the next-generation forest register should be, needless to say, prior consideration of updating the forest register data is also needed. It is necessary 

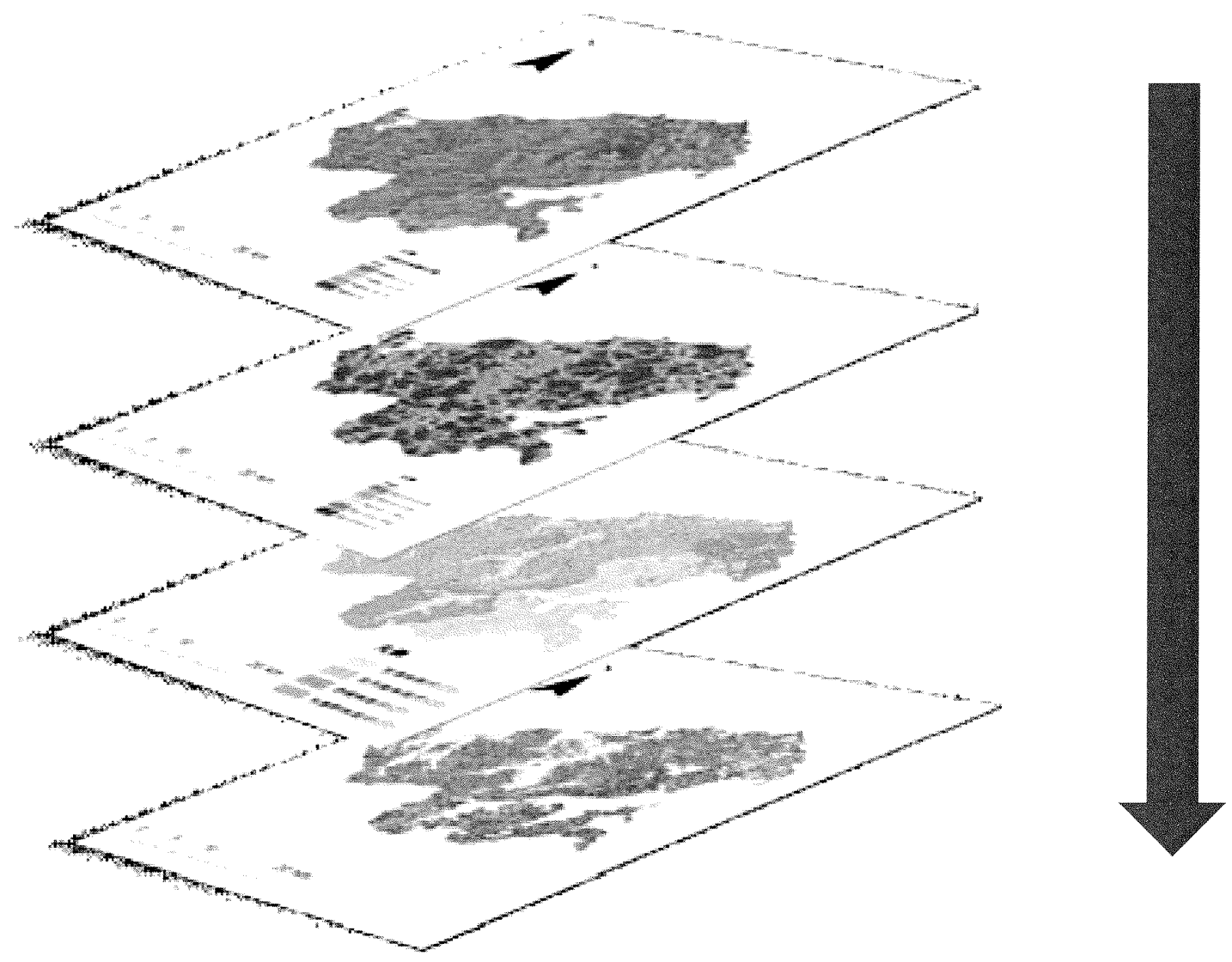

Fig. 1 Image of overlay

The GIS creates different geographic information for every thematic map and conducts spatial analysis dealing with these maps as a layer. From the top, reclassified inclination map, distance sectional map from forest road, administrative district map, age class map of plantations in the area of Tango Promotion Administration Office in Kyoto Prefecture. By using overlay function, we can visualize the geographical advantage condition of each forest stand.

and important to set up fewer steps for manual data updating. In this section, we will consider future ways to obtain GIS data. We have already seen the five development phases of the forest GIS, and since the first and second-generation forest GIS uses drawing data, polygons can be used. GIS in the newer phases, however, are provided with different data obtaining methods.

The third-generation forest GIS uses remote sensing data. Remote sensing data is raster data, so the spatial analysis with GIS means raster data analysis in many cases. Additionally, the analysis results will be integrated into sub-compartment polygons when necessary.

The fourth-generation forest GIS has a system where various observations or research results are analyzed and it also provides a function to distribute the analysis results to relevant local people through the Internet, and the observation or research results basically consist of point data with some GPS information in the future. The major method of analysis using the GIS will be the calculation of planes from points, utilizing the spatial analysis function.

The fifth-generation forest GIS utilizes external digital geographic information in various formats for policy making.
This means the system uses not only the data from a database created by the users. You may realize that the establishment of a database consisting of many items is not so significant in this sense. Additionally, since the proper external responsible division will update each external database, people concerned with forests and forestry should discuss what types of data they update as the forest GIS data.

As mentioned above, the fundamental purpose of the GIS is to conduct spatial analysis of multiple thematic maps. The thematic maps used for the analysis must have a reliable source and assured accuracy and reliability, and the users should take responsibility to update the data provided by them. Most data updating according to ground surveys will basically use point data with the GPS information, and planes calculated with points by using the spatial analysis function will be released to other divisions.

\section{DIVISION OF THE FOREST REGISTER}

Forests functions in a way that provide public benefits, and such information should be open to the public, in principle. Various forest conservation activities such as 
model forest movements have been developing in many places throughout the country, and by sharing the forest information, mutual understanding with stakeholders should be encouraged and forest planning which involves ordinary citizens should be established. Municipal forest improvement plans, in particular, require the disclosure of forest information in order to improve transparency and accountability. Since the forest register includes some personal information, however, it has not been disclosed yet.

Prefectural governments supervise the forest register, and its contents slightly vary depending on prefectures. Some prefectures have nearly 200 items registered in the register. However, it is thought that only a small part of the items are related to personal information, although this is not for certain because the register is not open to the public. In other words, since the forest register partially contains personal information, information that has nothing to do with personal information is also not disclosed.

In order to solve this problem, the current forest register information should be divided into different groups. Considering relationships with the forest GIS, it makes sense to divide the forest register information according to the contents of multiple thematic maps. One of those thematic maps will be about forest owners, which contains the personal information. It is appropriate for the thematic map containing personal information and its attribute information not be open to the public.

Division of the forest register according to thematic maps makes it easier to access needed data when users analyze information with the forest GIS, and additionally, it enables them to set up different data updating systems and timing according to the thematic map. Also, updating forest management data can be committed to forestry cooperatives or manufactures in the log production industry, and updated data can be collected in an electronic format.

\section{WHAT WILL THE NEXT-GENERATION FOREST REGISTER BE LIKE?}

Now we can envision the outline of the next-generation register centered around the GIS. When the forest GIS is applied to policy making or consensus building, related information should be collected from inside and outside the organization, and geographic information should be processed with the GIS's spatial analysis function (Fig. 2). Accordingly, the fundamental form of the data consists of a thematic map and its attribute information. In addition, the spatial analysis function of the GIS can deal with various data formats of the thematic maps handled, including polygon data which has been used for the traditional forest GIS, raster data which can be obtained by remote sensing, point data which comes from field studies with GPS measurements, digital elevation models (DEM) and digital surface models (DSM) which are created through laser profiling from a plane. Information in the forest register is also divided into different sections according to multiple thematic maps, and each section of information is processed in the same way, with the spatial analysis function of the GIS. In terms of these methods, information in the forest register should be information that can be created or updated by people concerned with forests and forestry under their responsibility.

Since various thematic maps are used for the forest GIS as stated above, we need to think about what types of attribute information should be provided with the forest GIS as a minimum. The minimum attribute information may be ID numbers of sub-compartments created by prefectures and some other related information. The thematic map of the forest physiognomy sectional map, that is, forest stand map, should correspond to this.

Historical information can be treated in the following way from a practical point of view.

First of all, each thematic map needs to be placed on a proper position in the current of the time. Prefectural official forest information as statistical data can be a series of the thematic map as of April 1st, or October 1st of every year. A national census used for the demographic statistics is carried out on October 1st every five years, and the thematic map prepared on a specific day such as October 1st will be kept after the example of this. The thematic map of each year kept in this way becomes historical information used by prefectures and municipalities as administrative materials.

Secondly, there is another issue about each stand's historical information. To solve this issue, it might be practical to hyperlink it to the "Forest Karte (record)." Creating the Forest Karte with a spreadsheet program such as MS Excel helps because the users can use another sheet for a new fiscal year. Some advanced forestry cooperatives already create the Forest Karte, so using hyperlinks will be a better way to respect their formats and contents. Accordingly, the forest GIS extract stands that the users want to see by searching data, and detailed historical information of each stand can be accessed through hyperlinks. In other words, detailed historical information of every single stand is not kept on the forest GIS, but the forest GIS just narrows down and extracts a certain group of stands by a search condition, according to this manner of use.

The remaining issue is about the treatment of historical information about each stand. This is about the historical information on forest land; for example, the current artificial forest land has been an artificial forest land for a long time, or it used to be a natural forest. The accumulation of thematic maps on a specific day such as April 1st enables us to use such information in the future, but ideally, we have to prepare such information on past situations using the results of aerial photographic analyses. In the case of a mountain disaster, wind damage, and snow damage, a damage map should be prepared for all such occasions. Eventually, the accumulation of historical information on stands in 10-meter mesh would be good enough.

\section{NEED FOR FORESTRY EXPERIMENT STATION-TYPE FOREST GIS}

We have seen a draft proposal of the next-generation forest register based on the GIS, and its basic concept is that forest information is created, managed, and updated on a basic thematic map basis. Here, basic thematic map means the thematic map of basic data. It solves issues and meets 


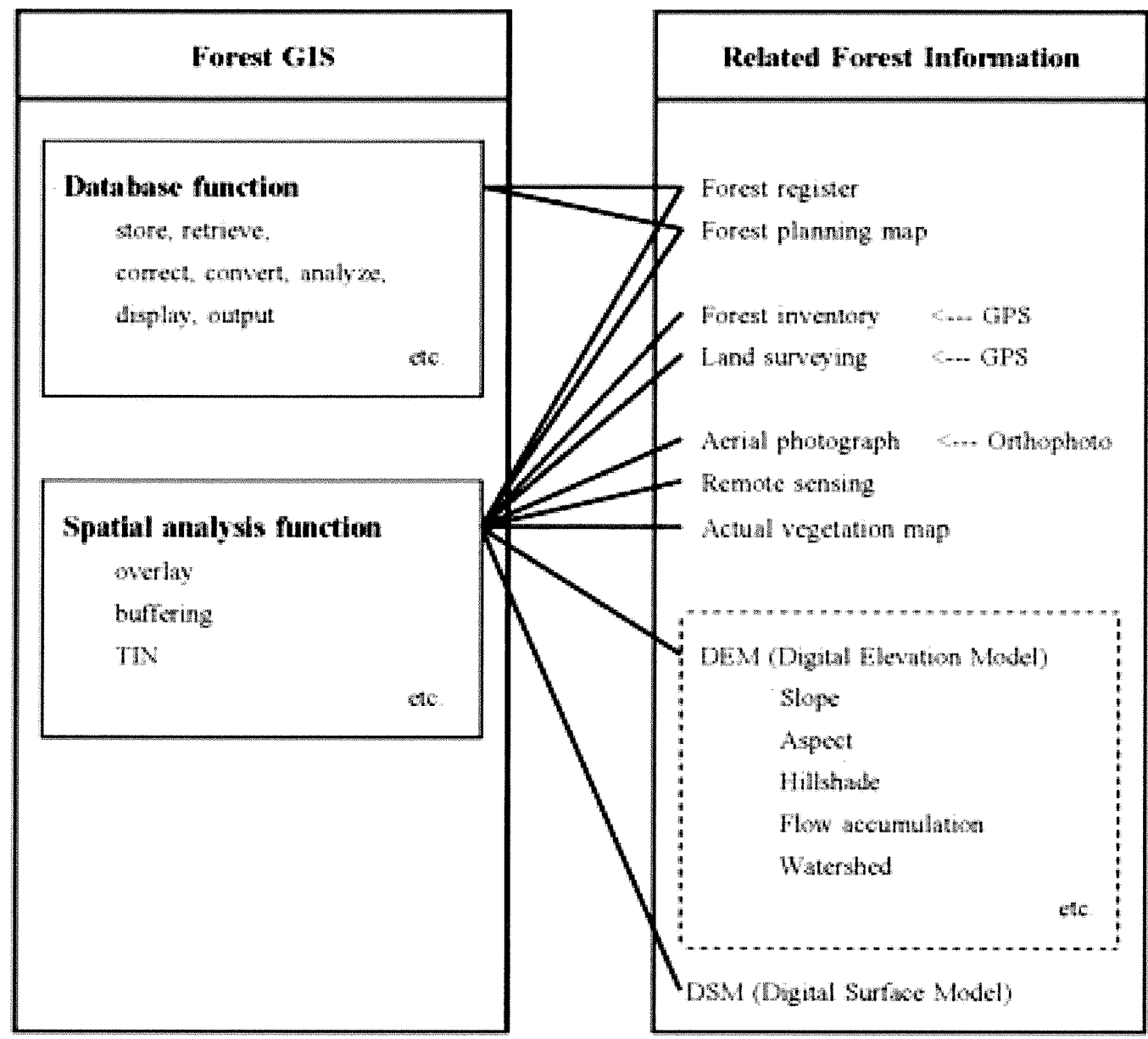

Fig. 2 Functions of forest GIS and related forest information

When the forest GIS is applied to policy making or consensus building, related information should be collected from inside and outside the organization, and geographic information should be processed with the GIS's spatial analysis function.

requests by using the spatial analysis function of the forest GIS to pick and use various thematic maps at the same time. Such a flexible structure enables the forest GIS to be developed as desired according to various needs.

However, groups of specialists of the highly advanced spatial analysis are required because administrative offices and forestry cooperatives cannot have a perfect command of the advanced spatial analysis function of the GIS. In this section, we call this kind of forest GIS which conducts advanced analyses "forestry experiment station-type forest GIS."

A forestry experiment station-type forest GIS is equipped with advanced spatial analysis functions, and analysis and research results obtained from the full use of these functions are provided to the forest GIS used by administrative offices and forestry cooperatives. This means this type of GIS is expected to create and provide new forest GIS information in each region, using the latest IT technologies such as remote sensing, GPS, and the Internet.

As social needs for local forest information increase, it is thought that only technical experts at forestry experiment stations can analyze various types of forest information collectively, conduct scenario analyses based on expected future events, and create geographic information which is suitable for applying to policy making and consensus building. Proper facilities and groups of experts who have advanced knowledge should be established or developed immediately.

People concerned with forests and forestry do need some IT help and support for their business because they have to deal with complicated, diversified and extensive nature, and the forest GIS is an indispensable system. Also, the forestry experiment station-type forest GIS is expected to provide the newest forest information in a proper way.

\section{CONCLUSION}

The next-generation forest register should be developed based on the assumption that it is used for the forest GIS, and consequently, the forest register information should be divided into multiple pieces according to thematic maps and their attribute information should be managed separately. This means the forest GIS is used as a tool for spatial analysis, 
not a database. The administrative data is an accumulation of thematic maps created every year. Data on forest management should be shared with forestry cooperatives and updated by forestry cooperatives electronically. The issue of personal information can be resolved by dividing the forest register information and managing it separately. Detailed historical information of stands is not necessarily contained in the database; rather, utilizing hyperlinks added to the Forest Karte improves the flexibility of the Forest Karte. The most important thing in the improvement based on the PDCA cycle is originality and ingenuity of on-site workers, so the management of the Forest Karte is entirely left up to onsite workers. Although data updating is always a significant issue to any system, the method of separately managing forest registries enables us to establish a system of taking responsibility of data updating for each thematic map.

This structure of the next-generation forest register can reduce the number of functions of the forest GIS used by onsite workers and the number of tasks they perform. This is because they only have to download the forest register information they need in a thematic map format. When they want new thematic maps or new data, they can ask an expert of the forestry experiment station-type forest GIS to create such new items. Although we call this system forestry experiment station-type forest GIS, they can ask proper external consultants to take care of this instead.

As I've mentioned repeatedly, I'm against the idea of creating a huge database consisting of so many items as a next-generation forest register. Creating such a database requires a great deal of labor, and additionally, updating the data is also laborious work. In addition, a database with an inflexible structure may have quite a bit of trouble when used in the future.

One of the basic requirements for the next-generation forest register is the management of forest information on a thematic map basis by using the spatial analysis function of the forest GIS. I hope these ideas are a starting point for considering the next-generation forest register.

\section{LITERATURE CITED}

TANAKA, K. and Yoshida, T. (2005) Forest GIS. (In Nagoya University ICCAE and JICA-Chubu (2005) GIS Technology for Sustainable Management of Natural Resources and Agricultural Products). 121-132, Textbook of the JICA GIS Training Course

(Received 16 September 2012)

(Accepted 29 January 2013) 